\title{
Situación actual de la integración del Diseño en las empresas manufactureras del Cantón Pelileo para la gestión creativa y de procesos productivos de la indumentaria en denim
}

\section{Current situation of the integration of Design in the manufacturing companies of the Pelileo Canton for the creative management and productive processes of clothing in denim}

María Cristina Paredes Morales

Universidad Técnica de Ambato, Ecuador

Jorge Luis Santamaría Aguirre

Universidad Técnica de Ambato, Ecuador

Efraín Marcelo Pilamunga Poveda

Universidad Técnica de Ambato, Ecuador

Autor para correspondencia: mc.paredes@uta.edu.ec,jl.santamaria@uta.edu.ec, em.pilamunga@uta.edu.ec

Fecha de recepción: 26 de septiembre de 2018 - Fecha de aceptación: 15 octubre de 2018

Resumen: Este estudio se enfoca en identificar estrategias que permitan integrar al diseño en las empresas manufactureras de indumentaria en denim del Cantón Pelileo, provincia de Tungurahua. Para ello se reconocen factores que impiden y facilitan la integración de esta disciplina en las mismas; usando el estudio de caso como metodología, la investigación examina la situación actual a nivel país y del sector empresarial en general del Cantón (macro entorno) y de las empresas en estudio frente al diseño (micro entorno). Mediante un análisis PESTEL se pone en evidencia las características del contexto, en cuanto a los factores políticos, económicos, sociales, tecnológicos, ecológicos, legales y demográficos; y la herramienta FODA permite visualizar la situación y percepción de las empresas en estudio.

Palabras Claves: gestión del diseño; empresas manufactureras; integración; estrategias

Abstract: This study focuses on identifying strategies that allow the integration of design in denim clothing manufacturing companies in the Pelileo Canton, Tungurahua province. For this, factors that impede and facilitate the integration of this discipline in them are identified. Using the case study as a methodology, the research examines the current situation at the country level and the business sector in general of the Canton (macro environment) and the companies under study against design (micro-environment). Through a PESTEL analysis, the characteristics of the context are highlighted, in terms of political, economic, social, technological, ecological, legal and demographic factors and the SWOT tool allows to visualize the situation and perception of the companies under study.

Key Words: design management; manufacturing companies; integration; strategies 


\section{Introducción}

A nivel mundial, muchas empresas han entendido la importancia que tiene el diseño y lo han adoptado dentro de sus estrategias para alcanzar ventajas competitivas y diferenciadoras, como alternativas para poder responder a un mercado con una gran proliferación de productos y consumidores que desean aumentar su calidad de vida.

Powell (2014) afirma que las empresas que mejor lleguen a entender el rol que juega el diseño en los procesos de innovación, más harán uso del mismo para alcanzar diferenciación y una competitividad sostenible. Por otro lado Ariza et al., (2007) manifiesta que las empresas se ven en la necesidad de posicionarse mejor, tener respuestas rápidas a los cambios y al crecimiento de la demanda; además, recalca que el consumidor posee características exigentes a la hora de su compra, razón por la cual las empresas deben reorientar sus esfuerzos para ir más allá de satisfacer las necesidades por medio de la incorporación del diseño como una herramienta estratégica, (pág. 21).

El nivel de especialización de los procesos que se ejecutan dentro de una empresa cada vez requiere de actividades de investigación, diseño, manejo de materiales, servicio al cliente, es decir, la multidisciplinariedad es una realidad. En consecuencia "la faceta del diseño ya no es una parte opcional de la estrategia empresarial y comercial, sino que debe ser su esencia”, (Peters, 2005, pág. 448). Ahora bien, el diseño podrá consolidar su rol solamente a través de un proceso bien gestionado.

A nivel internacional las Micro, Pequeñas y Medianas Empresas (MIPYMES) no tienen una comprensión del rol y desarrollo de la Gestión de Diseño, ya que no lo utilizan de manera consciente, sistemática o estratégicamente, (Magallanes., 2014, pág. 15), lo que genera un estancamiento y a la vez cierre de muchas organizaciones.

Las empresas manufactureras de indumentaria en denim del Cantón San Pedro de Pelileo en Ecuador, no son la excepción, por lo que la importancia de la investigación se enfoca en la necesidad de instrumentar acciones con el objetivo de apoyar directamente a la empresa para contribuir a su entorno económico, establecimiento, crecimiento y consolidación. Mediante el método de estudio de caso, se estudian cuatro empresas previamente seleccionadas de la totalidad, toda vez que fue necesario que las mismas tengan un diseñador trabajando dentro de su organización o un departamento de diseño para finalmente dar a conocer los alcances del diseño desde la evolución de sus fundamentos y cómo éste se puede convertir en un recurso para que la participación de este tejido empresarial crezca de manera significativa y sostenida en el país, caso contrario, algunas empresas se mantendrán de forma débil en el mercado, otras desaparecerán y el diseño no alcanzará su desarrollo que debe tener dentro de la economía nacional.

\section{Metodología}

La investigación se centra en el método de estudio de caso por la naturaleza del trabajo, ya que tiene un enfoque cualitativo. Éste método es una herramienta valiosa porque permite medir y registrar la conducta de las personas involucradas en el fenómeno estudiado, así como 
también la obtención de la información en base a un abanico de fuentes tanto cualitativas como cuantitativas.

"Los estudios cualitativos son amigables de adaptarse al estudio de caso generalmente, por tratarse de temas que son únicos y que tienen determinadas características que ameritan un estudio profundo y un acercamiento más real del contexto donde se desarrolla el fenómeno a investigar", (Chaves \& Weiler, 2016, pág. s/p).

El estudio partió con una revisión bibliográfica, de donde se obtuvo bases teóricas entorno a la gestión del diseño, así como también la revisión de informes para identificar la situación del macro entorno en base a los factores políticos, económicos, sociales, tecnológicos, ecológicos y legales que influyen en el sector en estudio. Además por medio de entrevistas se realizó el FODA de las empresas seleccionadas.

\section{Convergencia disciplinar: Gestión y Diseño.}

Existen múltiples perspectivas de la gestión del diseño, que tienen un recorrido desde el año 1965 hasta la actualidad. Esto refleja un amplio grupo de personas en el mundo académico, los sectores público y privado, el mundo de los negocios y la industria, la profesión del diseño o los organismos públicos y gubernamentales involucrados en éste ámbito (Best., 2007, pág. 12). López (2016) identifica tres grupos de definiciones de la gestión del diseño que se resume:

Tabla 1 Enfoques de la gestión del diseño

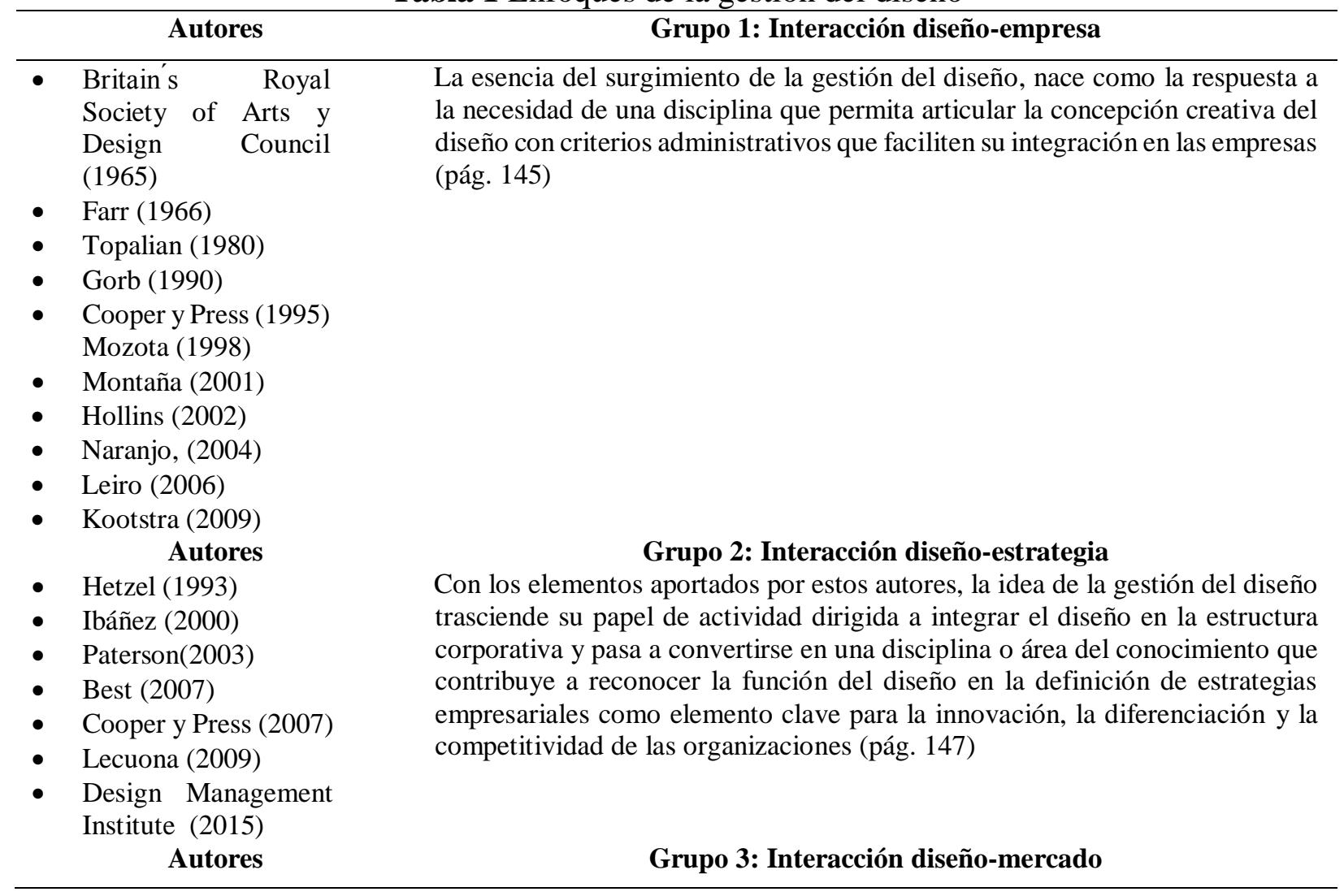


- Willock (1981)

- Ughanwa y Baker (1989)

- $\quad$ Blaich (1993)

- Finizio (2002)
Suponen una mirada externa para la identificación de oportunidades de mercado y el logro de ventajas competitivas. Esto supone la realización de una serie de acciones hacia el exterior mediante la adopción de políticas de diseño que permitan gestionar eficazmente el diseño para dar respuesta a los desafíos competitivos del mercado y lograr una identificación exitosa de las expectativas y necesidades de los clientes y consumidores (pág. 149).

Fuente: López, M. (2016, págs. 144-147)

Desde estas visiones, se evidencia al diseño como un valor que no se puede eludir dentro de las estructuras organizativas empresariales, si se desea que los productos- servicios sintonicen con las demandas reales del mercado. Además, asume un rol amplio al vincular varias áreas del sistema productivo, lo que le da un enfoque interdisciplinario que integra en un solo lenguaje todas las actividades de la empresa.

En vista que el diseño ha extendido su horizonte protagónico dentro del escenario contemporáneo, debido a que en las sociedades van surgiendo problemas con diferentes características. Determina que los fundamentos del diseño no sólo se enmarcan en el "cómo producir", sino "qué producir", "para quién producir" y "por qué producir", lo que ha provocado que se genere la conexión disciplinar de saberes y se traslade a un diseño estratégico.

Según Bermúdez (2008), el diseño estratégico más que un término constituye un método que permite acercarse a las necesidades específicas del entorno a través de una aproximación a los aspectos sociales, culturales y políticos de la sociedad para detectar problemáticas y a la vez mirar oportunidades desde una visión interdisciplinaria por lo que no se puede adoptar respuestas globales para un mismo problema.

Esto muestra, que la forma tradicional de concebir el diseño desde un punto estético y proyectual ha quedado atrás ya que ahora formar parte integral del proceso conceptual, estratégico y productivo de muchas empresas, considerándole como un factor clave en los procesos de innovación.

\section{Macro entorno: Sector manufacturero de textil y confección del Ecuador}

El sector manufacturero de textil y confecciones ha constituido desde su nacimiento como uno de los sectores que ha impulsado el desarrollo económico de un país. Involucra diversos aspectos que se integran en cuanto a recursos naturales y humanos (empleo).

La industria textil en el Ecuador, es una de las actividades más antiguas y tradicionales. Sus inicios se remontan en la época colonial, donde la Sierra Centro Norte - que actualmente es Ecuador- se experimentó el auge de la exportación, (Varela, 2011, pág. 3). Desde entonces, el sector se ha desarrollado a tal punto que según el Censo Nacional Económico 2010, está comprendido por tres grandes actividades productivas: manufactura, comercio y servicios. (Manuel, Muñoz, Durán, \& Mayiya, 2016, pág. 4), por lo que se le define como "el sector de la economía que produce telas, hilos, ropa y productos relaciones, dentro de los cuales se incluye el calzado", (Alencastro, 2016, pág. 2). 
Sin embargo, ésta actividad ha tenido que enfrentar grandes desafíos de alzas y bajas porque no ha sido considerado como una industria de prioridad para el desarrollo del país. Éste sector se caracteriza porque demanda mano de obra intensiva, lo que le convierte en una fuente importante de generación de empleo. (Varela, 2011).

Dentro del registro histórico, las empresas textiles ecuatorianas han dirigido sus ventas en su mayoría al mercado local y en un menor porcentaje a mercados extranjeros. Sin embargo, a partir de la década de los 90, las exportaciones textiles fueron incrementándose, salvo por algunas caídas en los años 1998 y 1999. A partir del año 2000, momento en que el Ecuador toma su moneda nacional al dólar, se genera un incremento en las exportaciones del $8.14 \%$ en contraste al año 1999, siendo el año 2013 donde alcanza el record de exportaciones textiles, superando los 114 millones de dólares (Varela, 2011).

Por otro lado, según Manuel, A. et al (2016), la distribución del sector se encuentra dividido en varias actividades económicas, siendo éstas de manufactura, comercio y servicio. La fabricación de prendas de vestir, excepto prendas de piel abarca el 74,2\% de la totalidad sector, siendo una cifra representativa.

\section{Análisis Macro entorno y Micro entorno}

Las empresas al encontrarse frente a un entorno en constante cambio, es de suma importancia se direccionen estratégicamente para sobrevivir. Gracias a un estudio estratégico del entorno externo e interno las organizaciones consiguen comprender y estudiar la situación real y actual de la empresa, para así definir una ruta clara que posibilite la formulación de estrategias dentro de sus posibilidades, teniendo en cuenta sus recursos propios y capacidades para finalmente poner en marcha la estrategia más factible y adecuada para la propia empresa.

\section{Análisis PESTEL}

La herramienta de análisis PESTEL se ha convertido en una técnica de análisis estratégico con el objetivo de ayudar a las organizaciones a alinearse de la mejor manera con lo que sucede en su entorno. Conocer el contexto donde se desenvuelven las empresas permite fundamentar, identificar y prever los cambios que se pueden dar alrededor de la realidad actual y un comportamiento futuro. Es de suma importancia tener claro los cambios del medio, ya que éstos puedan producir un impacto favorable (oportunidad) o adverso (amenaza); sin embargo, las empresas no están en la capacidad de cambiar el entorno, pero si pueden diseñar un plan estratégico que les permita aprovechar los aspectos positivos del entorno y poder enfrentar los negativos para que se vuelvan sostenibles y mejorar su posición competitiva, (Córdova., 2005). En base a esto se presenta un registro de datos relevantes del contexto ecuatoriano dentro de aspecto político, económico, social tecnológico, ecológico y legal.

Tabla 2 Oportunidades y amenazas del macro entorno

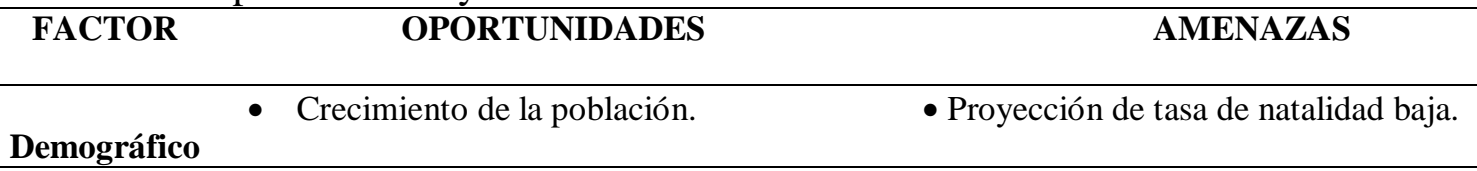




\begin{tabular}{|c|c|c|}
\hline Políticas & $\begin{array}{l}\text { - Estructura productiva que supere la } \\
\text { exportación de la materia prima. } \\
\text { - Generar trabajo y empleo dignos. } \\
\text { - Promover la productividad, } \\
\text { competitividad y calidad de los productos } \\
\text { nacionales. } \\
\text { - Mejorar encadenamientos productivos. } \\
\text { - Diversificar la producción nacional con } \\
\text { pertinencia territorial. } \\
\text { - Vincular los sectores: público, productivo } \\
\text { - } \text { y las universidades. } \\
\text { - Fomentar la producción nacional con } \\
\text { - Fortalecer y fomentar la asociatividad. } \\
\text { - Eliminar las tasas arancelarias. } \\
\text { - Fortalecer cadenas locales: enfatizando } \\
\text { - } \text { Or el proceso y no solo en el producto. } \\
\text { - Orientación exportadora }\end{array}$ & $\begin{array}{l}\text { - Corrupción. } \\
\text { - Deterioro Institucional. } \\
\text { - Desestabilidad Política }\end{array}$ \\
\hline Económicos & $\begin{array}{l}\text { - } \quad \text { Crecimiento del PIB. } \\
\text { - Tasa de desempleo bajo. } \\
\text { - Proyección de interés descendente. } \\
\text { - Exportaciones no petroleras han } \\
\text { mejorado. }\end{array}$ & $\begin{array}{l}\text { - Tasa de inflación descendente. } \\
\text { - Tasa de interés alto para las Pymes. } \\
\text { - Historial crediticio negativo } \\
\text { - Incremento de la deuda pública. } \\
\text { - El índice de confianza empresarial } \\
\text { decreciente. } \\
\text { - El índice de competitividad decreciente. } \\
\text { - Ecuador es un país difícil para hacer } \\
\text { negocios. } \\
\text { - Disminución de la producción } \\
\text { manufacturera dentro del sector textil y } \\
\text { confección. } \\
\text { Confianza del Consumidor es } \\
\text { decreciente. }\end{array}$ \\
\hline Social & - Sociedad consumista. & $\begin{array}{l}\text { - } \text { Estructura social compleja y rígida. } \\
\text { - } \text { Conformismo. } \\
\text { - Sujeciones de ciertos grupos. } \\
\text { Paternalismo. } \\
\text { - Sociedad ahorradora. }\end{array}$ \\
\hline Tecnológicos & $\begin{array}{l}\text { - Mejorar en la gestión empresarial } \\
\text { - Mejorar en el proceso productivo } \\
\text { - Mayor velocidad de producción con la } \\
\text { implementación de la tecnología. } \\
\text { - Incremento en inversión de las TICS. }\end{array}$ & $\begin{array}{l}\text { - Países Asiáticos los que tienen el mayor } \\
\text { desarrollo del sector. } \\
\text { - Implementación de prácticas desleales } \\
\text { en países orientales. } \\
\text { - Cambio en la demanda de productos } \\
\text { - textiles. } \\
\text { - Las innovaciones en maquinaria. } \\
\text { - Grandes inversiones. }\end{array}$ \\
\hline
\end{tabular}

Fuente: Elaboración propia

\section{Análisis FODA}

Este análisis permite visualizar el cuadro de la situación actual de una empresa u organización, que conlleva a un diagnóstico de las fortalezas, oportunidades, debildiades y amenazas para la toma de decisiones acordes con los objetivos y políticas de las mismas. Mediante las conclusiones del estudio, los empresarios pueden mirar la situación de su propia 
institución y del mercado en el que ésta se desenvuelve, lo que mejorará la competitividad de las estrategias de mercadeo y ventas que se diseñen. Es por esto que para el diagnóstico de las empresas en estudio se efectúo esta técnica teniendo los siguientes resultados.

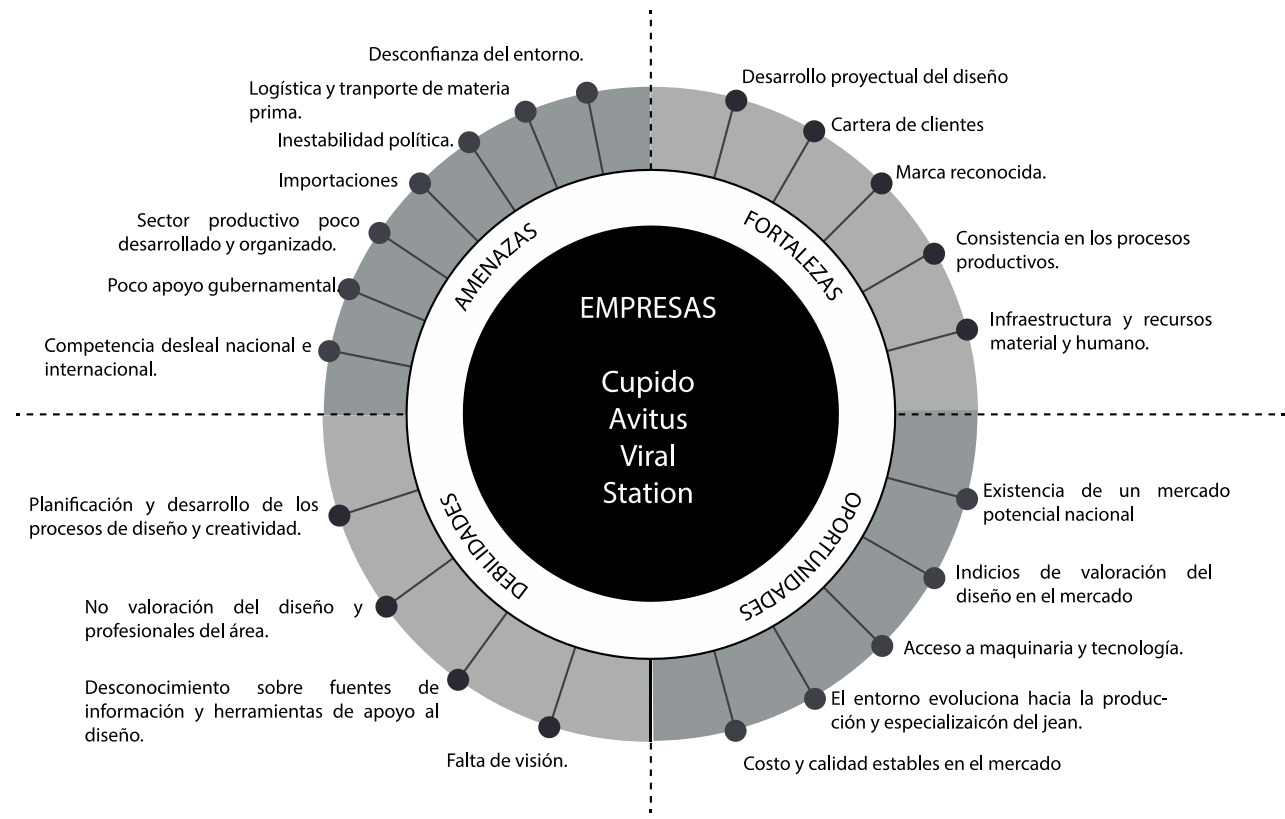

Figura 1: Resultados del análisis FODA

\section{Resultados}

Según Best (2007), una estrategia de diseño se enfoca en la forma que la empresa desea hacer uso de los recursos del mismo así como sus procesos, con el objetivo de satisfacer mejor las necesidades operativas de la empresa. Las estrategias de diseño permiten que la política de la empresa se vincule, ya que esta permite generar normativas que van a "apoyar el proceso y coordinan la información sobre la planificación y las diferentes directrices de diseño para cubrir las necesidades de todos los agentes implicados" (pág. 49).

Las empresas de manufactura de indumentaria en denim del Cantón Pelileo que han sido parte del estudio han empezado a implementar el diseño como parte de ellas, sin embargo, estás reflejan necesidades más amplias de diseño para poder seguir desarrollándose y ser más competitivas, como se reflejan en los diferentes análisis que se ha desarrollado.

Para lo cual, mediante la información obtenida del análisis PESTEL y el FODA se han identificado tanto las oportunidades- amenazas (macro entorno) del primero y las debilidadesfortalezas (micro entorno) del segundo. Estos dos métodos han permitido identificar y desarrollar proactivamente las oportunidades de diseño traducidas en estrategias, toda vez que permiten ver la relación que tiene la empresa con su mundo exterior (Best., 2007, pág. 30).

Bajo esta postura, y después de realizar el análisis de la información obtenido en la investigación se plantean estrategias generales con el fin de que puedan aplicarse en diferentes contextos, de lo cual se tiene lo siguiente: 
Tabla 3 Estrategias de mercado.

\begin{tabular}{lll}
\hline \multicolumn{1}{c}{ Factores } & & \multicolumn{1}{c}{ Estrategias } \\
\hline - $\begin{array}{l}\text { Un mercado potencial nacional. } \\
\text { Sociedad desconfiada, sin hábitos } \\
\text { de consumo. }\end{array}$ & $\bullet$ & $\begin{array}{l}\text { Enfoque hacia el público consumidor. } \\
\text { diseño. }\end{array}$ \\
& & Cambio en la percepción del mercado consumidor con \\
& & respecto al producto nacional. \\
& - & Incentivo al consumo de productos nacionales. \\
& - & $\begin{array}{l}\text { Entorno productivo con reglas claras y apoyo de las } \\
\text { autoridades. }\end{array}$
\end{tabular}

Fuente: Elaboración propia

En base al análisis PESTEL y FODA se identificó que una de las oportunidades que tienen las empresas es un gran mercado nacional potencial, al cual se debe conquistar conociéndolo mediante estudios que permitan identificar los requerimientos, necesidades, gustos, valores de forma real.

Además, se debe recalcar que el consumidor ecuatoriano presenta un nivel de desconfianza ante el producto nacional, lo que ha provocado que no desarrollen hábitos de consumo con pertenencia y a la vez adquieran productos extranjeros. Ante esto, mediante el desarrollo de productos con características avanzados se puede lograr añadir un mayor valor agregado a través del diseño.

\section{Acciones}

- Estudios de mercado para determinar las necesidades y características propias del consumidor nacional.

- Desarrollo de productos con características funcionales que cubran los requerimientos del consumidor.

- Desarrollo de campañas de promoción para fomentar el interés hacia los productos nacionales.

- Vinculación de los sectores productivos con el gobierno municipal y provincial mediante mesas de diálogo donde se integren profesionales del diseño para el apoyo y desarrollo de nuevos productos.

Tabla 4 Estrategias de infraestructura.

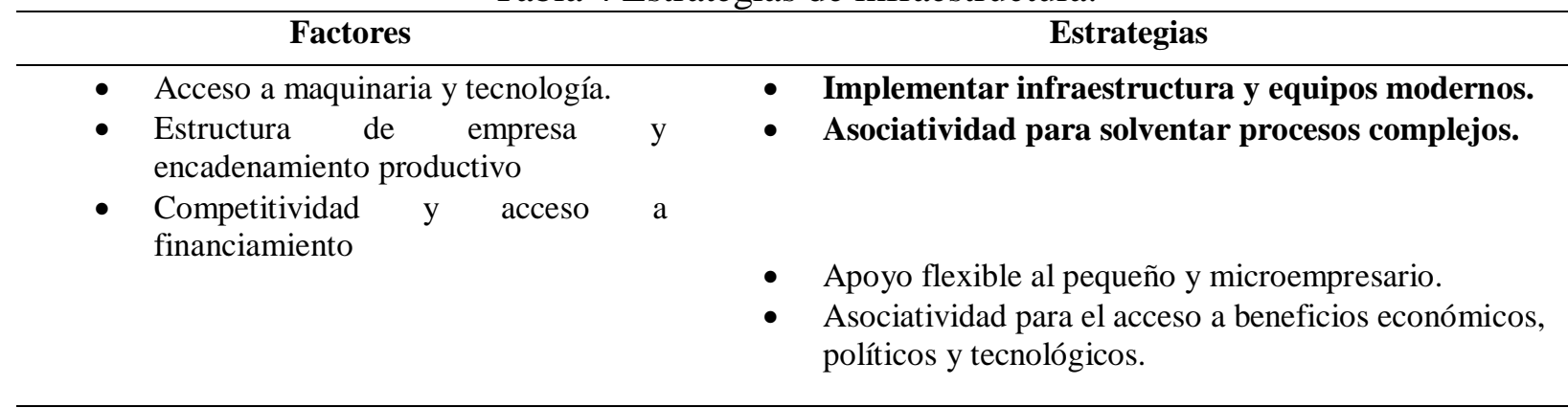

Fuente: Elaboración propia

Las políticas de salvaguardias para el acceso a maquinaria y tecnologías para los sectores productivos del Ecuador representan una oportunidad para las empresas porque por medio de 
financiamiento crediticio y la construcción de asociaciones sólidas como puentes de acceso a estos beneficios permitirá a las empresas implementar recursos materiales que apoyen a las actividades de diseño.

\section{Acciones}

- Buscar financiamiento a través de las asociaciones.

- Desarrollo de proyectos para la implementación de servicios complementarios desde donde el diseño puede apoyarse que complementen la actividad productiva por medio de las asociaciones.

- Organización de las asociaciones en busca de proyectos económicos crediticios que apoyen a sus miembros y al desarrollo de los otros proyectos antes mencionados.

Tabla 5 Estrategias de desarrollo de producto.

\begin{tabular}{|c|c|}
\hline Factores & Estrategias \\
\hline $\begin{array}{l}\text { - } \begin{array}{l}\text { Desarrollo proyectual del diseño } \\
\text { (productividad y valor agregado) }\end{array} \\
\text { - } \begin{array}{lll}\text { Procesos, tecnologías y la } \\
\text { academia. }\end{array} \\
\text { - } \begin{array}{ll}\text { Potenciales afectaciones al } \\
\text { producto, empresa y mercado. }\end{array}\end{array}$ & $\begin{array}{l}\text { - } \text { mejora cualitativa, valor agregado a través del diseño. } \\
\text { - } \text { programas de apoyo y capacitación para la diversificación } \\
\text { de los productos y mejora de los procesos desde la } \\
\text { academia. } \\
\text { - Fomentar una cultura de consumo que valore el diseño. } \\
\text { - Implementar la gestión de procesos creativos y desarrollo } \\
\text { de nuevos productos a través del diseño. }\end{array}$ \\
\hline & $\begin{array}{l}\text { - Cambio en la percepción del mercado consumidor con } \\
\text { respecto al producto nacional. } \\
\text { - } \quad \text { Incentivo al consumo del producto nacional. } \\
\text { - } \quad \text { Planes enfocados en el potencial productivo territorial. } \\
\text { - } \quad \text { Estrategias diferenciadores para mercados globales. }\end{array}$ \\
\hline
\end{tabular}

Fuente: Elaboración propia

Las empresas han podido desarrollar la parte proyectual del diseño, es decir que las capacidades del diseño solamente se ha centrado en otorgar características estéticas al producto. Este aspecto los empresarios lo aprecian como una fortaleza, sin embargo, como menciona la teoría, el diseño tiene otros alcances que sobrepasan los límites tradicionales bajo los cuales se ha venido desarrollando. Para poder incluir al diseño, es necesario implementar la gestión de los procesos creativos conjuntamente con el desarrollo de nuevos productos, mediante personas capacitadas que le otorguen la formalidad estratégica al diseño.

\section{Acciones}

- Proyectos de diseño que den origen a nuevos productos y nuevas líneas de productos.

- Desarrollo de programas de capacitación en: cultura empresarial, imagen y comunicación, creatividad y desarrollo de productos, procesos productivos y calidad, análisis de tendencias, materiales, nuevas tecnologías.

- Vinculación entre profesionales del diseño y empresarios para compartir experiencias y desarrollar proyectos.

Tabla 6 Estrategias de consumo.

Factores Estrategias


- Cartera de clientes.

- Producción territorial.

- Sociedad desconfiada, sin hábitos de consumo.
- Recuperar la confianza mediante productos funcionales antes que ornamentales.

- Experiencia del consumidor que genere valor agregado al producto.

- Fomentar el crecimiento de nuevos productos con denominación de origen.

- Enfoque hacia el público consumidor.

- Diversificar los segmentos en función a los nuevos perfiles de consumidor.

- Valorar estratégicamente la calidad y la pertenencia por sobre la estrategia de costos y volumen.

Fuente: Elaboración propia

Las empresas cuentan con la fortaleza de tener una cartera de clientes estable para la venta de sus productos. Por otro lado tienen la oportunidad de que en el territorio donde se encuentran instaladas se ha desarrollado una estructura amplia para la producción. En contraste a esto, existe un mercado que aún no ha sido conquistado, para lo cual es importante recuperar la confianza del consumidor mediante campañas de promoción, experiencias enfocadas en los requerimientos del mercado. Sin embargo los puntos positivos deben ser perfeccionados mediante una capacitación integral del funcionamiento de una empresa tomando como base al diseño como el corazón de los entornos empresariales.

\section{Acciones}

- Desarrollo de campañas de promoción para fomentar el interés hacia los productos nacionales.

- Desarrollo de programas de capacitación en: cultura empresarial, imagen y comunicación, creatividad y desarrollo de productos, procesos productivos y calidad, análisis de tendencias, materiales, nuevas tecnologías, etiquetado.

- Vinculación entre profesionales del diseño y empresarios para compartir experiencias y desarrollar proyectos.

- Generación de marcas propias que comuniquen la pertenencia a la zona y también la protección legal de la imagen de la región.

- Genera experiencias positivas a través de productos funcionales y a través de espacios efímeros (tiendas, exposiciones, ferias, entre otros) para la comercialización de los productos.

Tabla 7 Estrategias de organización y comunicación interna.

\section{Factores}

- Infraestructura, recursos materiales y talento humano.

- $\quad$ Estructura de empresa y encadenamiento productivo.

- Organización y comunicación interna de la empresa.

\section{Estrategias}

- Capacitación en cultura corporativa y comunicación organizacional.

- Concientización en el uso de recursos y mejor manejo de residuos. 
- Competitividad que vaya de la mano con profesionales y operarios capacitados en actividades específicas que den valor agregado al producto.

- Asociatividad para el acceso a beneficios económicos, políticos y tecnológicos.

- Asociatividad para solventar problemáticas endémicas de la región y el sector productivo.

- Un gobierno comprometido con el pequeño y mediano productor, reflejado en políticas tangibles.

- Estructuras de empresa optimizadas para la mejora productiva.

Fuente: Elaboración propia

Las empresas con el tiempo han ido implementando una infraestructura y recursos para las actividades de diseño, sin embargo en lo que respecta a la organización y comunicación interna de las mismas no se lo realiza de una manera efectiva. Como parte de los resultados del estudio se ha podido detectar que al ser empresas creadas más por tradición y herencia, no cuenta con una respectiva organización formal como empresa, es decir que no han intervenido profesionales capacitados. Esto justifica las falencias que presentan las empresas del territorio. Para lo cual es importante que se desarrollen capacitaciones en cultura corporativa y comunicación organizacional tanto a los directivos como a los profesionales de diseño.

Además, al ser la producción de indumentaria en denim una actividad que se caracteriza por el uso de procesos de lavandería, las empresas se enfrentan a una problemática ambiental que deben afrontarla de manera consiente en el uso de recursos y el mejor manejo de residuos.

\section{Acciones}

- Desarrollo de programas de capacitación en: cultura empresarial, imagen y comunicación, creatividad y desarrollo de productos, procesos productivos y calidad, análisis de tendencias, materiales, nuevas tecnologías, etiquetado, uso de recursos y medio ambiente.

- Generación de marcas propias que comuniquen la pertenencia a la zona y también la protección legal de la imagen de la región.

- Desarrollar una autoevaluación del sector productivo y de cada empresa para identificar la realidad en la que se encuentran y mejorar sus estructuras organizacionales.

- Organización de las asociaciones en busca de proyectos económicos crediticios que apoyen a sus miembros y al desarrollo de los otros proyectos antes mencionados.

- Vinculación de los sectores productivos con el gobierno municipal y provincial mediante mesas de diálogo donde se integren profesionales del diseño para el apoyo y desarrollo de nuevos productos.

Tabla 8 Estrategias con enfoque legal y social.

Factores Estrategias

- Marca reconocida

- Responsabilidad social y ecológica

- Propiedad intelectual
- Comunicación y campañas de marca.

- Capacitación, concientización en el buen manejo y beneficios de la marca.

- La marca como una vía de acceso a nuevos mercados. 
- Fomentar el crecimiento de nuevos productos con denominación de origen.

- Protección y comunicación de marca.

Fuente: Elaboración propia

En la actualidad la identidad del producto expresado por medio de la marca, es muy importante ya que el bien material debe tener presencia y características propias en el mar de las demandas que existe en el mercado. Por lo cual, la generación de marcas propias que comuniquen la pertinencia territorial mediante estudios apoyados de la academia permitirán crear un espacio con huella nacional en el contexto global.

\section{Acciones}

- Generación de marcas propias que comuniquen la pertenencia a la zona y también la protección legal de la imagen de la región.

- Desarrollo de campañas de promoción para fomentar el interés hacia los productos nacionales. - Contratación de servicios profesionales para la comunicación y manejo de la marca.

Tabla 9 Estrategias de planificación del diseño.

Factores Estrategias

- Planificación y desarrollo de los procesos de diseño y creatividad.

- Políticas de diseño

- Mejor posicionamiento ante producto extranjero y de contrabando.
- Inclusión del diseño en la cadena de valor de la empresa.

- El diseño como eje transversal en el proceso multidisciplinario productivo de la empresa.

- El diseñador como catalizador de la innovación.

- Estudios enfocados a las características, necesidades y cualidades propias del mercado ecuatoriano.

- Búsqueda de soluciones reales y no la adaptación desde otros mercados.

- $\quad$ protección y cuidado del producto nacional.

Fuente: Elaboración propia

Uno de los factores que afectado al diseño dentro de las empresas, es que éstas no tengan definidas políticas de diseño lo que ha provocado que la planificación y desarrollo de los procesos de creatividad y por ende del diseño no sea llevado a cabo. Razón por la cual es necesario incluir al diseño en cada uno de los eslabones de la cadena de valor de la empresa y del sector mediante la cohesión de profesionales del diseño especialistas en diferentes áreas del diseño.

\section{Acciones}

- Vinculación entre profesionales del diseño y empresarios para compartir experiencias y desarrollar proyectos.

- Implantar modelos de negocio y estructuras productivas que integren y prioricen al diseño en sus procesos de desarrollo de productos.

- Contratación de servicios profesionales para la gestión de los procesos creativos y proyectuales de diseño. 
- Protección de la identidad y de los productos derivados de los procesos de diseño de las empresas para el cuidado del producto nacional.

- Desarrollar una autoevaluación del sector productivo y de cada empresa para identificar la realidad en la que se encuentran y mejorar sus estructuras organizacionales

- Planteamiento de modelos de desarrollo adaptados a la realidad del sector productivo de las empresas locales.

\section{Conclusiones}

De acuerdo a los resultados obtenidos de cada una de las empresas, se evidencia una inmediata integración del diseño en niveles más altos, ya que las mismas al estar situados en un territorio con un amplio tejido productivo de indumentaria en denim y formar parte de un mercado totalmente globalizado, necesitan implementar estrategias a corto plazo enfocadas al cambio en su filosofía empresarial, la imagen corporativa y el trabajo conjunto con profesionales en las diferentes áreas de diseño. Estos puntos constituyen la plataforma de arranque para que posteriormente todos los otros aspectos pueden ir desarrollándose en función al diseño y a la vez las empresas puedan obtener ventajas competitivas que les permita mantenerse en el mercado.

Las estrategias planteadas en función al diseño conllevan a la creación de políticas de diseño dentro de las empresas, ya que éstas generan el ambiente adecuado que apoye al buen desempeño de las actividades de diseño. 


\section{Bibliografía}

Ariza, R., \& Ramirez, R. (2007). Herramientas para mejorar la gestión del diseño en PyMEs. Jornadas de Innovación y Desarrollo, 6.

Peters, T. (2005). El círculo de la innovación. Amplíe su camino al éxito. Barcelona : Editorial Deusto.

Magallanes., D. G. (2014). Integración de la gestión de diseño en micro, pequeñas y medianas empresas.

Best., K. (2007). Design Management. Barcelona: Parramón.

López, M. (2016). Management and Desing: Convergence disciplinar.

Bermúdez, C. (2008). Innovación y diseño. Relevancia del Diseño Industrial en los Negocios INternacionales. . Conferencia Semestral del grupo de Investigación de Medio Ambiente y Diseño. .

Varela, M. (2011). Boletín mensual de análisis sectorial de MIPYMES: Ropa deportiva de algodón y polialgodón $(R l)$. Centro de Investigaciones Económicas y de la Micro, Pequeña y Mediana Empresa, Quito .

Manuel, A., Muñoz, F., Durán, G., \& Mayiya, I. (2016). Contrabando y su impacto en el sector textil ecuatoriano, durante el periodo 2010 - 2015. Revista Observatorio de la Economía Latinoamericana, Ecuador.

Alencastro, C. P. (2016). Análisis del sector Textil Ecuadtoriano 2009 - 2013. . Revista Científica ECOCIENCIA.

Córdova., M. (2005). Estudio de la situación actual de la empresa constructura Inmosolucion y su proyecto para mejorar la productividad. Quito.

Parada, P. (2015). Análisis PESTEL, una herramiento del estudio del entorno. Obtenido de http://www.pascualparada.com/analisis-pestel-una-herramienta-de-estudio-del-entorno/ wikipedia.

Villacís, B., \& Carrillo, D. (2012). Estadística Demográfica en el Ecuador: Diagnóstico y Propuestas. . Instituto Nacional de Estadísticas y Censos , Quito .

(15 de 04 de 2018). Recuperado el 15 de 04 de 2018, de Countrymeters: http://countrymeters.info/es/Ecuador

(07 de 2016). Recuperado el 15 de 04 de 2018, de Indexmundi : https://www.indexmundi.com/es/ecuador/poblacion_perfil.html

SENPLADES . (2017). Plan Nacional de Desarrollo Nacional 2017 - 2021-Toda una vida. 
Cámara de Comercio de Guayaquil. (2018). El impacto de las salvaguardias sobre la producción en el Ecuador. Departamento de Investigación y Proyectos, Guayaquil.

Lee, J. W. (1995). Capital goods imports and long-run growth. Journal of development economics. Ministerio de Industrias y Productividad . (2016). Política Industrial del Ecuador 2016 - 2025. Quito .

Sistema Nacional de la Calidad . (2018). Plan Nacional de la Calidad . Quito .

Kreab Gavin Anderson. (s/f). Una mirada a Ecuador: Una economía en evolución.

(30 de 03 de 2018). Recuperado el 25 de 04 de 2018, de Televistazo : http://www.ecuavisa.com/articulo/noticias/nacional/372936-ecuador-crecio-3-2017doble-lo-previsto

Rosero, J., \& J., S. (2006). Boletín No. 8: Desempleo en el Ecuador. Quito.

(10 de 07 de 2017). Recuperado el 25 de 04 de 2018, de El Comercio : http://www.elcomercio.com/actualidad/inflacion-cifras-ecuador-chile-brasil.html

Crónicas: las noticas del día. . (09 de 10 de 2017). Recuperado el 29 de 04 de 2018, de https://www.cronica.com.ec/informacion/item/20061-interes-por-ahorros-no-superan-el1-anual

Banco Central del Ecuador . (2018). Recuperado el 29 de 04 de 2018, de https://contenido.bce.fin.ec/documentos/Estadisticas/SectorMonFin/TasasInteres/Indice.h tm.

Cámara de Comercio de Quito. (2017). Boletín de Comercio Exterior. Cámara de Comercio de Quito.

Galindo, M. y. (2015). "Deuda Pública" en Serie de Estudios Económicos. . México DF: México ¿cómo vamos?.

Foro de Economía y Finanzas Públicas. (s/f). Análisis del manejo de las Finanzas Públicas y de la evolución del Endeudamiento Público durante el período 2000-2017. Foro de Economía y Finanzas Públicas.

El Universo. (18 de 01 de 2018). Nueva deuda por \$ 3.000 millones coloca Ecuador, a diez años plazo.

Mutis, G. (s/f). Confianza empresarial y competitividad.

Ecuadorinmediato. . (30 de 04 de 2018). Recuperado el 30 de 04 de 2018, de http://www.ecuadorinmediato.com/index.php?module=Noticias \&func=news_user_view \&id=2818833689 
Lall, S. (2001). "Competitiveness Indices and Developing Countries: An Economic Evaluation of the Global Competitiveness Report". World Development.

Cámara de Comercio de Guayaquil. (2017). Índice de Competitividad Global 2017-2018. Guayaquil .

Datosmacro. (2018). Obtenido de Ecuador - Doing Business : Facilidad para hacer negocios: https://www.datosmacro.com/negocios/doing-business/ecuador

Instituto Nacional de Estadísticas y Censos . (2018). Boletín Técnico IPI-M: Resultados Febrero No. 02 - 2018. Quito .

Morales, H. D. (2008). Índice de Confianza del Consumidor: Barómetro de Opinión de los Hogares Estadounidenses.

Banco Central del Ecuador . (2018). Indice de Confianza del Consumidor.

Quintana, J. (1971). El desarrollo de la Sociedad Ecuatoriana .

INEC . (2012). Principales Resultados: Encuesta Nacional de Ingresos y Gastos (ENIGHUR) 2011- 2012.

Líderes. (s/f). En el país se privilegia el consumo pero falta cultura de ahorro. Obtenido de http://www.revistalideres.ec/lideres/pais-privilegia-consumo-falta-cultura.html

Carregal, C. (2016). Análisis Tecnológicos y Prospectivos Sectoriales. Argentina .

Juárez, A. (2015). Análisis económico y financiero del sector textil en las comarcas centrales. Diagnóstico y recomendaciones. . Universidad de Valencia .

Instituto Nacional de Estadísticas y Censos. . (2014 ). Resumen Ejecutivo Módulo de Tecnologías de la Información y la Comunicación - TIC de las Encuestas de Manufactura y Minería, Comercio Interno y Servicios 2012-2013-2014. Quito .

Sustainable Brands Madrid. (18 de 02 de 2018). La industria textil y el medio ambiente. Recuperado el 06 de 05 de 2018, de http://sustainablebrandsmadrid.com/blog/la-industriatextil-y-el-medio-ambiente/

Rubio, V. (s/f). La Gestión ambiental en la Pequeña y mediana Empresa. Cámara de Comercio, Industria y Navegación de Castellón, Departamento de Industria y Medio Ambiente.

Palacios, A. (2017). Obligaciones y derechos tributarios que tienen los contribuyentes personas naturales obligadas a Llevar contabilidad pertenecientes a los segmentos de micro empresas y pequeñas empresas en el Ecuador. Pontificia Universidad Católica del Ecuador., Quito.

Constitución de la República de Ecuador. (2008). Quito, Ecuador. 
Dirección Nacional Jurídica. (2005). Código Tributario. Departamento de Normativa, Quito . El Telégrafo. (10 de 03 de 2018). Recuperado el 24 de 06 de 2018, de www.eltelgrafo.com.ec: https://www.eltelegrafo.com.ec/noticias/economia/4/la-declaracion-de-impuesto-a-larenta-2017-empieza-en-el-pais

El Comercio. (12 de 10 de 2017). ELComercio.com. Recuperado el 24 de 06 de 2018, de http://www.elcomercio.com/actualidad/impuesto-renta-ajuste-empresas-personas.html (2005). Código de Trabajo del Ecuador. Quito .

Ecuador - Índice de Percepción de la Corrupción. (s/f). Expansión/Datosmacro.com. Obtenido de https://www.datosmacro.com/estado/indice-percepcion-corrupcion/ecuador

Chaves, V., \& Weiler, C. (2016). Los estudios de casos como enfoque metodológico.

(s.f.). Recuperado el 23 de 04 de 2018, de Trading Economics : https://es.tradingeconomics.com/ecuador/gdp-growth 